\title{
Improving the accessibility of trust guidelines for junior doctors at North Bristol NHS Trust
}

Madelaine Tarrant, Calum Honeyman, Alex Aquilina, Katie Young

North Bristol NHS Trust

\begin{abstract}
Medicine is becoming increasingly protocol driven. This provides a standardised format for doctors to deliver best practice, especially in the acute setting.

40 junior doctors were asked to locate three Trust guidelines: venous thromboembolism (VTE) prophylaxis; antibiotic prescribing; and management of upper gastrointestinal bleed (UGIB). For each doctor the time taken and number of mouse clicks to access each guideline was recorded. Following successful redesign of the Trust intranet we completed a re-audit.
\end{abstract}

Initial results showed $48 \%$ of doctors were unable to locate the UGIB or the VTE guidelines within 5 min. For those who were able to locate the guidelines it took an average of $111 \mathrm{sec}$ and 17 mouse clicks. $100 \%$ of doctors were able to locate the antibiotic guidelines in 12 sec and with two clicks. These are accessible via a single port of access.

Following our redesign of the Trust intranet $100 \%$ of doctors located all three guidelines in an average time of 7.2 sec and in 2.1 clicks. Improvement in access to VTE prophylaxis and UGIB was statistically significant $(p=0.001)$.

Redesigning our Trust intranet homepage has significantly improved the accessibility of acute surgical and medical guidelines.

\section{Problem}

At North Bristol NHS Trust (NBT) there were concerns regarding the accessibility of Trust guidelines to junior doctors. Such a problem existed due to the complex structure of the intranet and the magnitude of guidance available. Junior doctors were struggling to locate useful guidelines in a timely manner and thus often not using them. This could have a direct impact on patient care, as it is these guidelines which enable the standardisation of medical practice and thus the delivery of highest quality care.

\section{Background}

Medicine has become increasingly protocol driven as this promotes best practice. Clinical guidelines, as defined by the American Institute of Medicine, are "systematically developed statements to assist practitioner and patient decisions about appropriate health care for specific clinical circumstances" . The use of guidelines and protocols is widely encouraged at NBT as these help to ensure high quality evidenced based medicine is delivered across the Trust. Not only do these promote patient safety but they can also be linked to reduction in malpractice litigation and to significant economic savings.

An example of guidelines improving care provision is the use of the venous thromboembolism (VTE) risk assessment tool. This is widely used across hospitals in the UK. These guidelines help doctors to assess and appropriately manage VTE risk in hospital inpatients. They have reduced VTE risk substantially.

Junior doctors, particularly when starting new jobs, rely heavily on guidelines and protocols. They often turn to these sources when in emergency settings or when they are lacking senior support. If these guidelines are not readily available they may not be used. This could contribute to a lack of standardisation and potentially lead to adverse outcomes.

NBT has a large number of evidence based guidelines that are available to all staff via the Trust intranet. Due to the wealth of information available, guidelines that are useful to junior doctors often get lost in the mix. In order to address this problem we set out to demonstrate the lack of accessibility of available guidance and to create a solution that would enable junior doctors to locate such guidance with ease.

\section{Baseline Measurement}

We challenged 40 junior doctors to find three key Trust protocols using the current intranet. Junior doctors were from FY1 (foundation year 1) to SPR (specialist registrar) and had worked at the Trust for varying lengths of time. The three protocols/guidelines were: VTE prophylaxis, management of upper gastrointestinal bleed (UGIB), and Trust antibiotic prescribing.

Doctors were asked to find each protocol in turn as quickly as possible. They had a maximum time limit of $5 \mathrm{~min}$. We recorded the number of seconds and number of mouse clicks on a set proforma. 
BMJ Quality Improvement Reports

The number of seconds would demonstrate time taken to locate each protocol and the number of clicks was used to give an indication of how direct the route to each protocol was.

The antibiotic guidelines can be found via a single point of access on the Trust homepage. All doctors successfully found these guidelines, and did so in an average time of $3.5 \mathrm{sec}$ and two mouse clicks. The UGIB and VTE guidelines required multiple steps away from the main homepage to locate. Forty-eight per cent of doctors were unable to locate these within the allocated $5 \mathrm{~min}$. Of the $52 \%$ who could locate them, it took an average of $111 \mathrm{sec}$ and 17 mouse clicks.

See supplementary file: ds3155.docx - "Young Tables and Figures"

\section{Design}

Our baseline measurement demonstrated a significant problem among trainees accessing guidelines that did not have a single point of access on the Trust homepage.

Our Trust intranet is home to over 100 Trust policies and guidelines. We surveyed junior doctors to find out which guidelines they felt were the most important to have easy access to. We collated the results and compiled a list of the top 15 guidelines most wanted by junior doctors.

Armed with our baseline results we approached the Trust's quality improvement (QI) lead and presented the problem. Our solution, a single portal for easy access to the top 15 Trust guidelines, was well received.

The next step was to locate all 15 guidelines within the existing intranet. We then developed a prototype intranet page that housed hyperlinks to these guidelines.

In order to ensure our page would be updated in future we arranged for the QI lead to take ownership of the page. We then worked with the webmaster who created a direct link from the homepage to our new site. The link was entitled 'useful guidelines for junior doctors'. From then on our guidelines would be accessible via a single mouse click from the homepage.

Following the development of our page we publicised its presence. This was achieved through: presentations at junior doctor teaching sessions; posters displayed throughout the Trust; an email to all junior staff; and finally a message on the Trust's 'message of the day' screen.

\section{Strategy}

Several weeks later, using our original proforma, we re-audited the accessibility of the initial three protocols. Again we recorded time in seconds and number of mouse clicks. After the completion of the exercise each doctor was asked for constructive feedback on the new page. We were interested in aesthetics, content, and ideas for future developments.

\section{Results}

In cycle one, $100 \%$ of doctors were able to locate the antibiotic guidelines in under $12 \mathrm{sec}$ (mean $3.5 \mathrm{sec}$, range 1-12 sec) and with two mouse clicks (range 1-12). In this pre-intervention cycle $48 \%$ of doctors were unable to locate the UGIB or VTE protocols within the allocated $5 \mathrm{~min}$. For the remaining $52 \%$ who could locate the guidelines they did so in an average of $45 \mathrm{sec}$ and 14 mouse clicks. It took a mean time of $60 \mathrm{sec}$ (range 8-201) and 11 mouse clicks (range 4-53) to locate the UGIB guidelines. The VTE guidelines took a mean of $149 \mathrm{sec}$ (range 12-240) and 16 mouse clicks (range 4-48).

In cycle two, post-intervention, all 40 doctors were able to find all three protocols within an average time of $7.2 \mathrm{sec}$ and 2.1 mouse clicks. As in cycle $1,100 \%$ of doctors were able to locate the antibiotic guidelines. The VTE prophylaxis guidelines were found in an average of $11.2 \mathrm{sec}$ and the UGIB guidelines were found in an average of $5.6 \mathrm{sec}$. The improvement in access to the VTE and UGIB guidelines was significant $(p=0.001)$ (see tables $1-3$ and figures 1 and 2).

\section{Lessons and Limitations}

In the development and implementation of our quality improvement project we have learnt a number of valuable lessons:

1. Our main challenge was finding a hospital body to take ownership of our proposed intranet page. As this was the first initiative of its kind there was no template to follow and as such we had to persevere and forge links across many departments including IT services and Trust headquarters. We were fortunate enough to liaise with the quality improvement and audit department which kindly took ownership of the page. This partnership had the added benefit of helping with website design and update.

2. Another obstacle was ensuring that our page would be kept updated in line with changes to Trust guidelines. As a solution to this problem we used hyperlinks to the existing guidelines on the Trust intranet. This means that when these guidelines are updated so too are our links.

3. An important lesson was to use all available resources to advertise our project to provide maximal benefit for our junior doctors. We learnt to use different methods such as: giving talks at the end of $\mathrm{FY} 1 / 2$ teaching; circulating a clear email highlighting our new site; placing posters in busy hospital areas and using the Trust 'message of the day'. The constructive feedback we received was invaluable in project development.

4. The success of the page has highlighted some new challenges. Many hospital departments are now looking to promote their guidelines on our site. We must be careful that significant expansion over the next year does not detract from the simplicity and clarity that allow easy access to guidelines when required the most.

5. We had a large QI group that often made the delegation and execution of tasks difficult to coordinate. To overcome this 


\section{BMJ Quality Improvement Reports}

we held regular meetings, had well-defined roles and used an online 'Google groups' spreadsheet to coordinate data collection.

6. For our project we looked at accessibility of guidelines for junior doctors. With the benefit of hindsight we could have included other health professionals who would also benefit from our site and have equal access to our intranet.

\section{Conclusion}

Junior doctors at NBT were not able to access hospital guidelines and protocols in a timely manner. By developing a new intranet page where all guidelines were found via a single port of access we were able to show huge improvements in accessibility of Trust guidelines and protocols.

Our next step is to continue to develop this site both in terms of aesthetics and content while maintaining its simplicity. This will be done under the guidance of team members staying on at the Trust and the QI and audit department. We promote the link to new junior doctors who start at the Trust each August by advertising it in their induction pack. This provides a useful reminder when on call.

\section{References}

1. Health Service in Clinical Practice Guidelines (1990). Clinical Practice Guidelines: directions for a new program. Washington, DC: National Academy Press.

2. American College of Obstetricians and Gynaecologists (2012). Standardisation of practice to improve outcomes. http://www.acog.org/Resources\%20And\%20Publications (accessed 5 Dec 2013).

3. Scottish Intercollegiate Guidance Network (2010). Prevention and management of venous thromboembolism. http://www.sign.ac.uk/pdf/sign122.pdf (accessed 5 Dec 2013).

\section{Declaration of interests}

Nothing to declare

\section{Acknowledgements}

Lucy Allanby, Nataly Gibson, Sheena Lam, Benjamin Plumb, Sarah Upperton, Katy Wells 